\title{
IN- AND CIRCUMSCRIBED SETS OF PLANES TO SPACE CURVES*
}

\author{
BY CLIFFORD BELL
}

1. Introduction. The problem dealing with in- and circumscribed polygons to plane curves has been studied extensively by Durège, $\dagger$ Sylvester, $\ddagger$ Story, $\S$ Cayley, $\|$ and others. The purpose of this paper is to investigate analogous problems for certain curves in 3 -space and for the cuspidal space curve of order $n+1$ in $n$-space. A construction similar to that used for the in-and-circumscribed polygon, using, instead of tangent lines, hyperplanes having contact of order $n-1$, gives what may be called an in-and circumscribed set of planes to the $n$-space curve.

2. Rational Quartic Curves in 3-Space. The quartic curves are the curves of lowest order that need be considered since the osculating plane to a twisted cubic does not intersect the cubic again.

Let $t_{k}$ be the parameter of the point at which the $(k-1)$ st osculating plane intersects the quartic and at which the $k$ th osculating plane has contact with the curve. It is evident that the necessary condition for an in- and circumscribed set of $n$ planes is that $t_{n+1}=t_{1}$. .

TheOREM I. There are no in-and-circumscribed sets of osculating planes to the cuspidal quartic.

Taking the equations of the cuspidal quartic in the form $x: y: z: w=t^{4}: t^{3}: t^{2}: 1$, it is found that $t_{n+1}=(-1)^{n} t_{1} / 3^{n}$. Hence the only solution of the equation $t_{n+1}=t_{1}$, in this case, is $t_{1}=0$, which gives no in- and circumscribed sets.

* Presented to the Society, November 29, 1929.

† Durège, Mathematische Annalen, vol. 1 (1869), pp. 509-532.

¥ Sylvester, American Journal of Mathematics, vol. 2 (1879), pp. 381-387.

§ Story, American Journal of Mathematics, vol. 3 (1880), pp. 379-380.

II Cayley, Collected Works, vol. 8, p. 212.

II In all cases only those sets that consist of two or more distinct planes are counted. 
ThEOREM II. The quartic having two distinct linear inflections has an infinite number of in-and circumscribed sets consisting of two osculating planes each and these are the only sets that exist for this quartic.

If the equations of this quartic are in the form $x: y: z: w$ $=t^{4}: t^{3}: t: 1$, we find that $t_{n+1}=(-1)^{n} t_{1}$, from which the theorem follows at once.

TheOREM III. The smallest number of osculating planes in an in-and circumscribed set to the quartic having a single linear inflection is three and the number of such sets is two.

If the equations of the quartic are $x: y: z: w=t^{4}: t^{3}:(t+1)^{4}: 1$, it is readily shown that

$$
\begin{aligned}
& t_{2}=-t_{1}\left(t_{1}+2\right) /\left(3 t_{1}+2\right) \\
& t_{3}=t_{1}\left(t_{1}+2\right)\left(t_{1}{ }^{2}-4 t_{1}-4\right) /\left[\left(3 t_{1}+2\right)\left(3 t_{1}{ }^{2}-4\right)\right] \\
& t_{4}=\frac{-t_{1}\left(t_{1}+2\right)\left(t_{1}{ }^{2}-4 t_{1}-4\right)\left(t_{1}{ }^{4}+16 t_{1}{ }^{3}-32 t_{1}-16\right)}{\left(3 t_{1}+2\right)\left(3 t_{1}{ }^{2}-4\right)\left(3 t_{1}{ }^{4}+12 t_{1}{ }^{3}-24 t_{1}{ }^{2}-48 t_{1}-18\right)} .
\end{aligned}
$$

Setting $t_{3}=t_{1}$, we find that $t_{1}=0,-1$, which give, respectively, the values $0,-1$ for $t_{2}$. Hence no in-and circumscribed sets of two osculating planes each exist for this quartic. The solutions of $t_{4}=t_{1}$ are $t_{1}=0,-1$ together with the roots of

$$
7 t_{1}{ }^{6}+28 t_{1}{ }^{5}-84 t_{1}^{4}-160 t_{1}{ }^{3}+80 t_{1}{ }^{2}+192 t_{1}+64=0 .
$$

The symmetry is such that the six solutions of this equation determine but two distinct sets.

Corollary. All the in-and circumscribed sets, consisting of three osculating planes to the quartic with a single linear inflection, are made up of real planes.*

THEOREM IV. The smallest number of osculating planes in an in-and circumscribed set to the rational quartic having no cusp nor linear inflection is two and the number of such sets is three.

The equations of this quartic are taken in the form $x: y: z: w$ $=(t+1)^{4}:(t+a)^{4}: t^{4}: 1$, where $a \neq 0,1$. Proceeding as before, we

* For the roots of equation (1) are all real. 
find that the solutions of $t_{3}=t_{1}$ are $t_{1}=0,-1,-a$ together with the roots of the equation

(2) $t_{1}^{6}+2(1+a) t_{1}^{5}+5 a t_{1}^{4}-5 a^{2} t_{1}^{2}-2 a^{2}(1+a) t_{1}-a^{3}=0$.

The first three values give the same values of $t_{2}$, and hence no sets are obtained from these roots. Equation (2) yields six different values of $t_{1}$, but because of symmetry we have only three sets of osculating planes.

Corollary. Only two of the sets of in-and circumscribed osculating planes to the rational quartic having no cusp nor linear inflection are real.*

3. The Cuspidal Curve of Order $n+1$ in $n$-Space. The parametric equations of the cuspidal $n$-space curve of order $n+1$ are $x_{1}: x_{2}: \cdots: x_{n}: x_{n+1}=t^{n+1}: t^{n}: \cdots: t^{2}: 1$.

THEOREM V. The locus of the point of intersection of an unclosed set of $n$ hyperplanes having contact of order $n-1$ with the $n$-space cuspidal curve of order $n+1$ is another $n$-space cuspidal curve of order $n+1$ having the same cusp as the first curve.

Let $t_{1}, t_{2}, \cdots, t_{n}$ be the parameters of the points of contact of the $n$ hyperplanes. The equations of the hyperplanes constructed at these points are

$$
\begin{aligned}
& a_{0} x_{1}+a_{1} t_{i} x_{2}+\cdots+a_{r} t_{i}^{r} x_{r+1}+\cdots \\
& \quad+a_{n-1} t_{i}^{n-1} x_{n}+a_{n+1} t_{i}^{n+1} x_{n+1}=0,(i=1, \cdots, n)
\end{aligned}
$$

where

$$
a^{r}=(-1)^{r}(n-r)(n+1) n(n-1) \cdots(n-r+2) / r \text { ! }
$$

and $t_{i}=t_{1} /(-n)^{i-1}$. Denote by $Y_{r}$ the $n$-rowed determinant obtained from the matrix

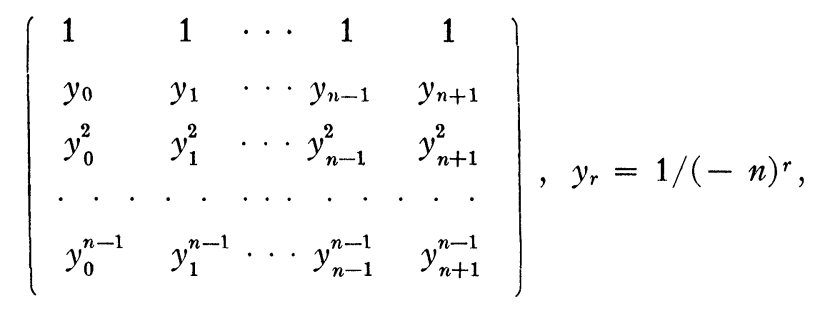

* For the last factor of (2) yields four real and two imaginary roots for all allowable values of $a$. 
by striking out the column having subscripts $r$. The solution of the simultaneous equations ( 3 ) is then

$$
x_{1}: x_{2}: \cdots: x_{n}: x_{n+1}=B_{0} t_{1}^{n+1}: B_{1} t_{1}^{n}: \cdots: B_{n-1} t_{1}^{2}: B_{n+1},
$$

where $B_{r}=(-1)^{r} Y_{r} / a_{r},(r=0, \cdots, n-1, n+1) . \quad B_{r}$ is never zero for any value of $r$, since the determinant $Y_{r}$ is equal to a product of the differences of the $y$ elements, all of which are different.* Hence the theorem is verified.

The number of unclosed sets that have their intersection point on the hypersurface $f\left(x_{1}, x_{2}, \cdots, x_{n+1}\right)=0$, of order $n^{\prime}$, is clearly $n^{\prime}(n+1)$. Call the first point of contact of the hyperplane with the curve $A_{1}$, the next $A_{2}$ and so on, the last intersection point being $A_{n+1}$, and distinguish between sets by the superscript. Thus $A_{k}^{i}$ stands for the $k$ th point of contact in the $i$ th set.

THEOREM VI. The points of contact or intersection points, $A_{k}{ }^{i}\left[i=1, \cdots, n^{\prime}(n+1)\right]$, with the $n$-space cuspidal curve of the unclosed sets of $n$ hyperplanes that have their intersection point on the hypersurface $f\left(x_{1}, x_{2}, \cdots, x_{n+1}\right)=0$, all lie on the hypersurface $f\left(c_{0} x_{1}, \cdots, c_{r} x_{r+1}, \cdots, c_{n-1} x_{n}, c_{n+1} x_{n+1}\right)=0$, where $c_{r}=B_{r}(-n)^{(k-1)(n-r+1)}$.

The parameters of the points $A_{1}{ }^{i}$ are given by the equation $f\left(B_{0} t_{1}^{n+1}, \cdots, B_{r} t_{1}^{n-r+1}, \cdots, B_{n-1} t_{1}^{2}, B_{n+1}\right)=0$. But $t_{1}=(-n)^{k-1} t_{k}$ and hence the parameters of the points $A_{k}{ }^{i}$ are given by $f\left(c_{0} t_{k}{ }^{n+1}, \cdots, c_{r} t_{k}{ }^{n-r+1}, \cdots, c_{n-1} t_{k}{ }^{2}, c_{n+1}\right)=0$, where $c_{r}=B_{r}(-n)^{(k-1)(n-r+1)}$. Hence it follows that the points $A_{\boldsymbol{k}}{ }^{i}$ all lie on the surface $f\left(c_{0} x_{1}, \cdots, c_{r} x_{r+1}, \cdots, c_{n-1} x_{n}, c_{n+1} x_{n+1}\right)=0$.

Thus it is seen that a simple magnification, dependent only upon $k$, deforms the given surface $f=0$ into one that contains all the points $A_{k}{ }^{i}$.

The University of California at Los Angeles

\footnotetext{
* See Dickson's Elementary Theory of Equations, 1914, p. 141.
} 